\title{
Vom Geist der Natur bei Paracelsus*
}

\author{
Heinrich Schipperges
}

Wenn vom Geist der Natur die Rede sein soll, von einem Geist der Natur auch des Menschen, dann denkt man unwillkürlich an das Licht der Natur und seinen glänzendsten Interpreten, an Theophrastus von Hohenheim, der sich später Paracelsus nannte. Als Arzt seiner Zeit - in einer Zeitenwende vertritt Paracelsus weder eine Heilkunde scholastischer Traditionen noch eine Medizin aufdämmernder Moderne, und schon gar nicht einen Eklektizismus im immerwährenden Streit der Fakultäten.

Paracelsus ist seinen eigenen Weg gegangen. Seine Medizin beruht auf einer höchst originellen, einer so eindeutigen wie eigenständigen Konzeption: auf einer Theorie der Heilkunde nämlich, die sich gründet auf jene «Philosophie der Natur», die dann allein auch den praktischen Arzt mit seiner heilenden Kunst zu bilden in der Lage ist.

Theophrast von Hohenheim wurde im Jahre 1493 an der Teufelsbrücke bei Einsiedeln geboren, und er starb - in der Blüte seiner Jahre - einsam und verarmt im Jahre 1541 in Salzburg. Dazwischen liegt ein unrastiges Wanderleben, das den jungen Arzt durch ganz Europa treibt: an die Hohen Schulen Italiens und an die Schmelzöfen der Bergwerke, über die Pilgerstrassen Spaniens wie auf die Schlachtfelder der Nordischen Kriege und in die Wirren der Bauernaufstände. Nach gescheiterter Praxis in Salzburg (1526) und kurzem akademischen Intermezzo zu Basel (1528) finden wir ihn abermals auf den Landstrassen, als fahrenden Arzt in nachgehender Fürsorge, als polemischen Laienprediger, als einen längst nicht gewürdigten Gesellschaftskritiker und Sozialreformer.

Fernab vom «bündigen Lehrgefüge» seiner Zeit rühmt sich Paracelsus mit wachsender Selbstsicherheit seiner einfachen, einer eindeutigen Sprache, da ich mich - wie er schreibt $(X, 199)^{*}$ - «keiner Rhetorik noch Subtilitäten rühmen kann, sondern nur der Zunge meiner Geburt und Landessprache, da ich bin von Einsiedelen, des Land's ein Schweizer, soll mir meine ländliche Sprache niemands verargen. Ich schreib' nicht der

\footnotetext{
* Guggenheim-Vortrag, gehalten an der Jahresversammlung der Schweizerischen Gesellschaft für Geschichte der Medizin und der Naturwissenschaften, Zürich, 24. September 1993.
} 
Sprache wegen, sondern von wegen der Kunst meiner Erfahrenheit». Wie hiess das doch so schön noch in Carl Spittelers «Olympischer Frühling»? «Lass nimmer deines Neides Krähenschwänze rauschen: / Ich heisse Paracels, mit keinem möcht ich tauschen!»

Seine so eigenständigen wie weittragenden Entwürfe sind freilich von den Zeitgenossen nicht aufgenommen worden, und sie wurden bald schon von den Nachfolgern weitgehend verfremdet. Bereits Toxites, der Sterzinger Arzt Michael Schütz, konnte 1574 konstatieren : «Es ist viel von Theophrastus ausgegeben, das nit ist.» Und auch Nicolaus Hunnius schreibt 1622 noch kurz und bündig: Er halte alle die Sache, die unter des Paracelsus Namen «in die mysticam philosophiam» gebracht wurden, samt und sonders für unecht, weil sie so gar nicht mit seinem «Schweitzerischen deutschen Stilum» übereinstimmten, so «dass ein jeder, der nur wenig Linien conferiret, verstehen kan, sie seyen beyderseits von einem Autor nicht entstanden».

Das ist ein klares Wort, das auch mich ermutigt, mich in dieser Feierstunde - Markus Guggenheim zu ehren und zu gedenken - ganz und gar zu konzentrieren auf das innere, das eigentliche, das so grossartige wie unverwechselbare Gedankengut des Hohenheimers, der von sich selber in aller Bescheidenheit gesagt hat: «Wer seinen Geist für den Heiligen Geist setzt, dessen Lust und Begehren ist, Nüsse zu knacken, die nicht geknackt werden wollen» (IX, 350).

Lassen Sie mich daher - und möglichst mit den markigen Worten des Paracelsus - nur die Wege aufsuchen zu jener vollen Wirklichkeit des Daseins, die sich im Licht der Natur erst erhellt, sich enthüllt nur im Walten der Zeit, um uns letztlich zu leiten in den Sinn der Natur und - so Paracelsus - «an das Letzte aller der Dinge», da, «wo jedes Ding allein in ihm selbst stehet und jubilieret in seiner Exaltation zu gleicher Weis' wie das Gold».

\section{Wege zur Wirklichkeit}

Beginnen wir zunächst mit den so abenteuerlich erscheinenden, dann aber immer mehr in das Licht der Natur gerückten Wegen, mit denen der Arzt Paracelsus die Ärzte seiner Zeit aus dem «Irrgarten», dem «Labyrinthus medicorum errantium» (1538) herauszuführen gedachte an das «Licht der Natur», in die volle Wirklichkeit mit ihren fünf Daseinsbereichen des gesunden wie kranken Menschen: seine Erbwelt und seine Umwelt, seine Zeit- 
gestalt und seine Mitwelt sowie seine letztgültige Verwurzelung in einem Absoluten, dem «Ens Dei».

Mit dem Bild vom Mikrokosmos wird der Mensch - mit seiner Natur, in seiner Geschichte, mit seiner Gesellschaft - leibhaftig in den konkreten Raum gestellt: wahrhaftig eine Welt an Erfahrenheit. Mit dem Wissen um diese Welt und im Geist dieser Natur erst ist die Voraussetzung gegeben für eine umfassende Welt- und Menschenkunde, eine wirkliche Medizinische Anthropologie: ökologisch orientiert, anthropologisch fundiert, sozial informiert und ethisch vertieft.

Wie aber kommt man - so hatte Paracelsus gefragt - heraus aus diesem Labyrinth, das uns ja nicht nur die verwirrende Pluralität der Weltbezüge repräsentiert, sondern auch die armseligen denkerischen Möglichkeiten eines Menschen mit seinem «viehischen Verstand»? Wie kommt man heraus aus dem dialektischen Gefängnis unserer «Hirnschale», los vom Gespinst der logischen Phantasien und rationalen Deduktionen? Wo haben wir denn noch ein Auge für die Welt, die Lesbarkeit dieser Welt? Wo finden wir auch da noch weiter, wo Kausalität und Induktion und die ganze formalistische Logik nicht weiterhelfen? Denn die Augen als solche verhelfen nur zum «Experiment», und eben nicht zur «Experienz» (XI, 92). Erst wenn Denken und Wahrnehmen zu einer Verbindlichkeit kommen, werden sie eine «scientia», werden wahres Wissen.

Bei diesem immer intensiver werdenden Umgang mit Szienz und Experienz aber, da erfährt man, wie viel später noch, aber aus gleichem Geist, der junge Goethe auf seiner «Reise in die Schweiz» (1797) notiert hat, «dass eine vollständige Erfahrung die Theorie in sich enthalten muss». Und noch einmal und gesteigert in den «Maximen und Reflexionen», wo es - so ganz paracelsisch - heisst : «Es gibt eine zarte Empirie, die sich mit dem Gegenstande innigst identisch macht und dadurch zur eigentlichen Theorie wird» - eine an Tiefsinn nicht auszulotende Bemerkung, die sich ihres Ranges durchaus bewusst zeigt, wenn Goethe schliesst: «Diese Steigerung des geistigen Vermögens gehört einer hochgebildeten Zeit an» - : Sie ist eben eine Sache des Niveaus!

Im hermeneutischen Gestaltkreis von «Theorica et Practica» erst erfährt man das Organ des erleuchteten Auges wie auch der begreifenden Hände, eine «vollkommene Erkenntnis», dass «die Hände greifen und die Augen sehen», um «das Ganze zu wissen und die Erkenntnis des Dinges, das den Glanz im Spiegel gibt» (VIII, 72). Diesen Glanz im Spiegel will Paracelsus beschreiben, will alles das zeigen, «was wiederum ganz wird und den Augen 
erscheint. Denn im Geist ist es nicht zerbrochen, sondern ganz» (II, 301). Diese Art von Erkennen im Geiste des Ganzen, auch «augenscheinliche Erfahrenheit» genannt, heisst bei Paracelsus «Erkennen im Licht der Natur»!

Die zunehmende methodische Einengung aller Wissenschaften von der Natur des Menschen, aber auch die methodologischen Möglichkeiten einer Erfassung des ganzen Menschen, des kranken wie des gesunden, sie lassen sich nirgendwo deutlicher aufzeigen als im Opus des Paracelsus. Hier lässt sich aber auch schon erschreckend deutlich die Schere erkennen zwischen dem zunehmend harten Kern der Naturwissenschaft und den zunehmend verblassenden Geisteswissenschaften, ein Zerfall in die «zwei Kulturen», und mehr noch : ein Bruch, der in der Neuzeit führen sollte zu einem verhängnisvollen Streit der Fakultäten.

Wie in einem überdimensionalen Koordinatensystem wird bei Paracelsus noch das einheitliche Bild des Menschen an der Welt sichtbar. Die Dinge der Natur kommen nicht nur zur Erscheinung, sondern auch zu ihrer Enthüllung. Der Kräuter Kräfte sind unsichtbar, und doch werden sie aufgespürt. Die Tiere bleiben stumm, und der Mensch bringt dennoch ihr Wesen in Erfahrung. Damit haben wir einen ersten Grund für das Bild der Welt, Bildung einer Welt aus dem Licht. Gibt nicht, fragt Paracelsus, das Gesicht den Augen einen recht guten Grund? Hat sich Gott nicht selbst mit Augen zu sehen gegeben? Und nun will sie ans Licht, diese Welt, und alle Wissenschaft dient allein dazu, «dass der gefangene Glanz frei werde». Das «Herz der Dinge», es will sichtbar sein, es soll sagbar werden. «Denn die Natur sieht in sich selbst, was in ihr ist und hat ein Auge, das in alle ihre Heimlichkeit sieht» (XII, 152). Nichts soll verborgen sein oder stumm bleiben, nichts ohne Erfahrung: «Es muss aller herfür, Geschöpf, Natur, Geist, Böses und Gutes, Aussen und Innen, und alle Künste und Doktrinen, Lehre und was immer geschaffen ist» (XIV, 131).

Das Licht erst macht offenkundig die Lichtung: Alle Natur, aller «augenscheinliche Verstand» (VIII, 70) der Dinge, will aus sich selber heraus sichtig und offenbarlich werden. «Denn wo ein Herz ist, da ist auch ein Mund, da ist auch eine Stimme, da ist nun die Erforschung des Herzens.» Das Auge, das ins Geheime der Natur geschaut hat, ist dem Munde gleich, der aus dem Herzen spricht. Im Auge liegen «die Künste des Suchens», die «Wege der Offenbarung», und sie alle wollen an das «Herz der Dinge» (XII, 155).

Was aber wäre das nun, diese Natur, von deren Geist hier so lichtvoll die Rede ist? Auch zu des Paracelsus Zeiten standen sich immer noch die beiden 
klassischen Naturphilosophien gegenüber, die mit den Namen des Platon und des Aristoteles gekrönt sind. Für die Platoniker liegt der sinnlich erfahrbaren Welt eine eher rationale Ordnung zugrunde, der «mundus intelligibilis». Natur als intelligible Ordnung manifestiert sich in der «machina mundi». Im Weltbild des Aristoteles hingegen gewinnen die natürlichen Dinge gleichsam «von selbst» Form; sie tragen den Ursprung von Bewegung in sich selbst, als eine «dynamis», die aus sich heraus auf Handeln (praxis) und Herstellen (poiesis) aus ist und so ihr Ziel in sich austrägt, in ihrer Vollendungsgestalt eben, der «entelecheia».

Beide Naturbegriffe haben augenscheinlich die Philosophie der Renaissance befruchtet; beide zeigen sich auch noch in der «neuen Wissenschaft», wo an die Stelle der «Idee» das «Gesetz» tritt, wo «Natur» ein Mittel in der Hand des Menschen wird, wo - wie Kant formuliert hat - «die Vernunft nur das einsieht, was sie selbst nach ihrem Entwurf hervorbringt», wo man folglich «die Natur nötigen müsse, auf ihre Fragen zu antworten, nicht aber sich von ihr allein gleichsam am Leitbande gängeln zu lassen».

Nun ist Paracelsus weder Platoniker noch Aristoteliker, noch gar ein frühreifer Kantianer; er will die Welt erfahren «nit nach aristotelischer Beschreibung, sondern nach der Beschreibung des Lichts der Natur» (In Ps. 103). Paracelsus hat und behält ein eigenständiges, eigenwilliges, ein höchst originelles Naturverständnis, das keineswegs der Herrschaft über die Natur dient, sondern eher der Orientierung in der Welt. Physis meint hier offensichtlich noch: Welt im ganzen (holon), aber auch jene Ordnung der Welt (taxis), wie sie dem Ursprung (arche) entstammt und wie sie aus ist auf das Eingebundensein (ethos) aller Dinge. Ethisches Verhalten aber kann sich nur auf eine Natur beziehen, die uns nicht nur vorgegeben ist, die uns auch aufgegeben ist.

Vielleicht ist der Mensch wirklich im Grunde ein eher technisches als natürliches Wesen, der «Fabrikator in die Figur», der «homo faber», ein «operarius», weniger freilich ein Macher als der «kybernetes», der Steuermann, wie schon Hippokrates den Arzt genannt hat, wie Isidor von Sevilla ihn als «moderator» umschrieb und wie Paracelsus ihn als «gubernator» benennt.

Auch unter dieser Intention sind hier unter dem Begriff «Natur» keineswegs die Gesetzlichkeiten eines unverrückbaren Kosmos gemeint und auch nicht die Beliebigkeiten einer evolutionären Entfaltung, sondern eher jenes dramaturgisch bewegte Geschehen, wie es uns im Urphänomen «Zeit» begegnet und die Paracelsus begreift als «Zeitigung», als «maturatio», als 
«Erwartung der Zeit». Aus einer solchen Natur, die Schöpfung meint wie Geschichte, kommen Gesundheit und Krankheit; aus ihr kommt nun auch die Arznei. Alle Funktionen des Leibes, sie tendieren auf eine fortschreitende Lebensentfaltung des Menschen, auf sein Wohl. Allzunahe Lebensziele werden dabei aufgegeben zugunsten fernerer, endgültiger; über das Naturhafte hinaus dominiert letztlich die Kultur.

Also will es der Geist der Natur! «Also wunderlich lehrt uns das Licht der Natur, durch die äussere Form das inwendige Herz zu erkennen - und hat diese Erkenntnisse in eine Kunst gesetzt», in die Heilkunst eben, die Lehre vom Heilen und vom Heile.

So hat dies Paracelsus formuliert in seiner so kühnen und elementaren Sprache und daraus gefolgert: Also ist die Natur des Menschen Arzt. «Denn so er der Natur hilft, so gibt sie ihm seine Notdurft und gibt ihm also zu eigen seinen Garten zu eigener Kultivierung. Denn so wir am gründlichsten allen Dingen nachdenken und trachten, so ist unsere eigene Natur selbst unser Arzt» (IX, 92).

Was aber meint das, dieser innere Arzt, diese Selbsthilfe der Natur als Selbstheilung? Nun, genau diese Frage hatte sich schon das Annebäbi im Jahre 1842 vorgelegt, in Jeremias Gotthelfs Roman «Annebäbi Jowäger» nämlich mit dem schönen Untertitel «... und wie es ihm mit dem Doktern ging». Da will die Bäuerin Annebäbi von ihrem Doktor ein Laxativum für ihren Sohn, und der Doktor hält das an sich so harmlose Mittelchen schon für viel zu gefährlich oder auch unnötig und rät: Da müsse man «die Natur machen lassen»; denn auch er, der Doktor, sei schliesslich nur «ein Diener der Natur». Genau da aber will Annebäbi es wissen, wer das wohl sei, «der Natur»! Und der eine meint, «der Natur», das sei ein Apotheker zu Bern, ein zweiter, das sei des Doktors Frau, und ein dritter glaubt gar, «der Natur», das sei der Teufel! Dass aber der Arzt nur Diener sei der Natur, davon hat Jeremias Gotthelf nicht lassen wollen.

\section{Heilkunst im Licht der Natur}

Wie aber zeigt er sich nun, in diesem «Licht der Natur», der «Geist der Medizin»? Wie gewahren wir die vielfältigen Stufen der Natur, die gerade in der Heilkunde auf ein Ganzes zustreben, auf eine höhere Einheit verweisen, auf das eben, was wir genannt haben den «Geist der Natur»? 
In den «Verteidigungsreden» seiner Kärntner Schriften, den berühmten «Defensiones», beschreibt Paracelsus, wie ursprünglich der «Geist der Natur» von Apollon, dem Heilgott, erleuchtet war, vom «spiritus Apollinis», und wie Gott von Anfang an dieses «Licht der Natur» hat wirken lassen «ohne einen befinsterten Geist». Nicht von einem Heiligen Geist sollen die Ärzte daher eine Antwort auf die Fragen ihrer oft so banalen Profession erwarten, sondern von jenem Licht der Natur, das nirgendwo anders sein kann als in eben dieser Natur.

Dass Gott letzten Endes auch «der Lehrer der Arznei» sei, das gilt nur unter dem Aspekt der ersten Schöpfung, nur «in der Weis' der Erschaffung». In ihrem Ursprung freilich, da waren alle Künste auf Erden göttlich, da ist und bleibt der Heilige Geist «der Anzünder des Lichts der Natur». Nun aber ist der Mensch auf sich gestellt, auf sein Opus an der Natur, berufen zur Bildung der Welt. Nunmehr geht alle Ordnung «aus der Art des Leibs, auch des Lichts der Natur». In dieser konkreten Ordnung ist der Leib gezogen und gewachsen, empfindet im Reifen «sein selbst» und wird immer weiter wachsen: «So ist die Art des Lichts der Natur»!

So lautet das in dieser alten, fremden, aber auch saftigen und bündigen Sprechweise, einem Denken und Sprechen, das oftmals mit Luthers Deutsch verglichen wurde, ein dennoch unvergleichlicher Wortlaut und Klangleib, auf den ich immer wieder zurückgreifen muss. Und lassen Sie mich auch jetzt einfach wieder einmal zurücktreten hinter diesen Sprachleib mit seiner so verdichteten Sprachkraft, die man nicht nachlesen kann, die man einfach sprechen und hören muss!

Die Philosophie soll im «Licht der Natur» derart gelehrt werden, so beginnt Paracelsus (und hier schon verschlägt es einem fast die Sprache vor der Fülle der Gesichte in diesem Kosmos Anthropos), derart : «dass in ihr der Mensch ganz erscheine und begegne, dass man in ihr finde alle Krankheiten und Zufälle, Gesundheit und Trübsal, alle Glieder und Gliedmassen, alle Teile und der Glieder Teilung, so viel am Menschen und im Menschen ist oder sein mag, und soviel man in der Natur sehen, wissen und erfahren kann, so viel vom ersten Menschen bis zum letzten einfallen mag oder eingefallen ist -, so ganz und vollkommen, dass auch die Augen, die Ohren, die Stimme, der Atem in der Welt gefunden wird, auch die Beweglichkeit, die verdauenden Organe, die austreibenden, die anziehenden und alles, was im Leibe not wäre zur Hülfe, zur Gesundheit, zu allen Dingen... Und so das alles auswändig an dir erfahren ist und du somit in diesen Dingen erfahren bist, alsdann geh' in den inneren Menschen» (VIII, 144). 
In diesem inneren Menschen aber, da ist nun die ganze Welt noch einmal, im Inneren ein Universum auch, ein kompletter Endokosmos und Mesokosmos. «Der nun also ein Philosophus ist, der soll alsdann in die Fakultät der Arznei treten und das Äussere in das Innere wenden. Das Umwenden gibt den Arzt, so aus der grossen Welt die kleine wird, eine derartige innere Richtung, dass er auf keine Weise mehr an dem inneren Menschen lerne, denn da ist nichts als Verführung und der Tod»(VIII, 86). Wer diesen Weg nicht geht, der bleibt ein blosser Experimentator, was besagen will: «ein Geratewohler und verzweifelter Hoffer» (VIII, 87) -, der ist eben kein Arzt, sondern «ein Verwalter des Glücks»!

So steht es mit der Philosophia, «die da trägt den Bau der Arznei» (VIII, 154)! Das gehört einfach zur Sache - so Paracelsus sogleich weiter -: «dass ein Arzt einen Menschen also lauter durchsehe, als durchzusehen ist ein destillierter Tau, in dem sich kein Fünklein verbergen mag, das nit gesehen werde. Und also durchsichtig soll er hineinsehen als durch einen quellenden Brunnen, wie viel Stein und Körner, mit was Farben und Formen sie sind, also offenbar sollen ihm sein die Glieder im Menschen auch. Dieselbigen Glieder soll er also durchsichtig haben wie der auspolierte Kristall, in dem sich kein Härlein möcht' verbergen. Das ist die Philosophei, auf die gesetzt ist der Grund der Arznei» (VIII, 71).

Philosophie hat hier sicherlich nichts zu tun mit den ausufernden Fachbereichen unserer Philosophischen Fakultäten; sie ist aber ebensowenig Naturkunde oder gar Theosophie, sie könnte am ehesten noch als Anthropologie verstanden werden, als eine Naturkunde vom Menschen. Und so auch die anderen Säulen der Medizin: die «Astronomia», die weder Himmelskunde ist noch Astrologie. Sie ist eine kosmologisch orientierte Zeitkunde und begleitet die pathogenetischen wie auch therapeutischen Prozesse. Und auch die «Alchimia» sollte nicht verwechselt werden mit banaler Goldmacherei und ebensowenig mit einer vorweggenommenen Biochemie, ganz zu schweigen von einem tiefenpsychologisch zu deutenden Individuationsprozess. Hier geht es eher um die biophysikalische Energetik eines Organismus und damit auch um den Sinn aller technologischen Prozeduren. Und «Physica» schliesslich, die letzte der vier Säulen, das wäre am ehesten noch das praktische Feld einer angewandten Naturphilosophie, wie es im ärztlichen Denken und Handeln wirksam wird und zur Meisterschaft kommt, zur «Virtus», wie manche Texte statt «Physica» sagen. Und auch dies mag als ein weiteres Symptom dafür gewertet werden, dass «Tugend» nicht anders als leibgebunden gedacht sein kann, dass «virtus» exakten physiologischen 
Bedingungen unterliegt, wie auch andererseits «gesundes Sein» konstitutiv bereits einer Wertung bedarf, jenes «Vorwalten eines oberen Leitenden» nämlich, wie Goethe «Geist» nannte.

Im Lichte dieser Natur erfahren wir erst wirklich, wie alles lebt und wirkt in tausenderlei Gestalten (XII, 372), wie die Handwerke blühn und viel tausend Künste: «Also triumphiert der Geist Gottes auf Erden unter den Menschen» (II/I, 145). Der Mensch erfreut sich im Licht der Natur und staunt und verwundert sich; er spielt in der Natur (XII, 254). Nichts darf feiern in diesem Spiel, ehe denn angebrochen ist die «güldene Welt», die wir jetzt schon zu kultivieren haben. Und wie Gott die Zerbrechlichkeit der Dinge in der grossen Welt arzneiet, also hat Gott auch dem irdischen Arzte zu wenden befohlen, zu reinigen, «aus welcher Reinigung der Mensch unzerbrüchlich hervorgehet wie Gold, ohne welche Reinigung aber nichts ist denn die täglich Zerbrechung. Diese Reinigung ist ein Werk wie das Feuer» (VII, 273). Sie ist das «opus magnum» aller Kunst!

Denn ein Arzt und Naturforscher muss - noch einmal ein grösserer Passus und in gleichem Tempo - «seinen universalischen Gang durch den ganzen Leib nehmen, zu gleicherweise wie die Sonn' die ganze Welt überscheinet und alle Äcker, Felder und Wiesen, Berg' und was darauf begriffen wird, ... und bekommt allen Kreaturen wohl, also dass sich alles ob ihr erfreuet. In solche Gestalt soll auch der Arzt in der Arznei beflissen sein, dass sie dermassen ein Durchscheinen sei des ganzen Leibs und zu gleicher Weis', wie auf Erden alle Kreaturen ihre Erquickung von der Sonne nehmen, ihre Fröhlichkeit und ihre Gesundheit». Und wie ein Sonnentag der Balsam aller Gewächse ist, so soll sich erweisen die Tugend der Arznei. «Und so ist die Arznei im Leibe wie die Sonne auf der Erde» (VII, 268).

In diesem so grandiosen irdischen Drama aber um Leben und Tod sollten Ärzte wie Patienten gleicherweise wissen, «dass Gott in den Krankheiten ebenso gelobt sein will wie in den Blumen des Feldes, obschon dies dem Menschen widerwärtig ist. Schaut aber an: Alle Vögel hat er geschaffen, das ist ihm ein Lob, auch die Würmer, Spinnen, Basilisken, sie sind ihm gleicherweise ein Lob wie die Nachtigall oder der Pfau oder vielerlei gut Gewächse wie Gold und Perlen, gleich wie auch viel Gift ob Arsenik oder Merkur-: es ist alles sein Lob! Also ist ihm zum Lobe, dass er uns die Gesundheit gegeben, also auch gleichermassen ein Lob ist die Krankheit; es ist eine Ordnung und ein Wesen» $(I, 327)$. In beiden sei zu sehen die gleiche Meisterschaft: im Zerbrechen wie im Ganzmachen. «Drum ist der Winter so löblich wie der Sommer. Beide wirken ein Werk. Daher ist alles ein Lob!» 
Das alles ist - wie Sie aus jedem seiner Sätze spüren werden - kein statisch abgeschlossenes Weltbild; das ist eher die stetig wachsende, in sich ausreifende Schöpfung, die uns an die Hand gegeben wird. Und so sind auch alle Heilmittel - sagt Paracelsus - «wie ein gewaltiger Himmel in der Hand des Arztes». Denn auch die Arznei ist «gerichtet gleich einem Schiff auf dem Meer, das keine bleibende Statt hat, sondern durch den Schiffmann geführt wird» (VII, 148), geführt und geleitet aber nicht nach dem gestrigen Wind, sondern nach dem Wind, wie er uns heut um die Ohren weht, heute und wie sehr erst morgen.

Darum so soll der Arzt wachsen, muss die Heilkunst in die Hände der Jungen gelegt werden. Denn - fragt Paracelsus sehr energisch - «wie wohl können die Alten noch wachsen? Sie sind ausgewachsen und verwachsen und im Moder vermösert und verwickelt, dass nichts denn Knorren und Knebel draus werden. Darum so ein Arzt auf einen Grund stehen soll, so muss er in die Wiege gesät werden wie ein Senfkorn und in derselbigen aufwachsen wie die Grossen bei Gott, wie die Heiligen bei Gott. Und sie müssen also wachsen, dass sie in den Dingen der Arzenei zunehmen wie ein Senfbaum, dass sie über alle wachsen. Solches muss mit der Jugend aufstehn und einwachsen. Wie wächst es denn noch ein in den alten Patres, die verwachsen sind und kommen daher, und die Zeit ist hin, haben nit geblühet, haben nit gesprosslet, haben nit ausgeschosst, sind nit im Märzen gewesen, wissen vom April nichts, wissen nit, ob der Mai blau oder grün ist, sind gekommen im Heumond - und nun wollen sie haben Frucht tragen?» (VIII, 212).

Wir bleiben zeitlebens die Arbeiter im Weinberg der Natur - es geht wohl nicht anders! Zur Bildung der Erde sind wir berufen, zum Lesen im Buch der Natur, das hier in keiner Weise mit mathematischen Lettern geschrieben, eine Lesbarkeit der Welt vielmehr, die nur mit ihren qualitativen Kräften gedeutet werden kann. Darin aber ist der Mensch nicht nur das Zentrum der Welt, sondern auch das aktive Glied in jenem anhaltenden Schöpfungsprozess, der uns die Wunder der Welt erst an den Tag bringt, die Erfahrung der vollen Wirklichkeit der Natur. Natur ist eben kein zeitenlos in sich ruhendes Gefüge, sondern eher eine «im natürlichen Lauf», im Reifwerden der Dinge wachsende Ordnung, wobei der Mensch nicht nur seinen eigenen Leib zeitlebens zu gestalten vermag, sondern mit seiner Technik auch die Welt da draussen, die grosse, wachsende, reifende Natur. Denn - so lautet einer seiner Schlüssel-Sätze - «die Schöpfung ist noch nicht zu Ende; der Mensch muss sie vollenden»! 


\section{Von der Verklärung der Welt im Licht der Natur}

Und damit kommen wir auf einen letzten Aspekt zu sprechen, den ich hier nur andeuten kann, den ich aber auch nicht aussparen darf, wenn schon vom Geist der Natur die Rede sein soll, und das ist des Paracelsus so weitgespanntes eschatologisch ausgreifendes Konzept von der Verklärung der Welt im Licht der Natur.

Mitsamt seiner Geschichte ist ja der Mensch eingespannt in ein grosses kosmisches Geschehen, eine gewaltige universelle Rhythmik, die auf ein Ziel zustrebt. Welt begegnet uns hier nicht nur als Erbwelt und Umwelt; Welt steht hier im Horizont der Zeit: als ein Miteinander möglicher Freiheit, als offenes System eigener Spielräume, Spielpläne, als ein dramatisch bewegtes biographisches Szenarium.

Das aber allein soll sein «die Art des Lichts der Natur», sagt Paracelsus, dass der Mensch wachsen und reifen soll in seiner unverkürzten Leiblichkeit. Denn «alle Dinge, die Gott geschaffen hat, die hat er in die Korporalität gleicher Prozesse ausgeführt» (III, 38). Gleichartige Substanzen aber bedingen gleichgerichtete Funktionen. Struktur und Funktion, sie bilden hier noch ein einheitlich geschlossenes, ein leibhaftiges Bedeutungskontinuum; sie zeigen uns die Ordnung der Natur. Und so ist denn für Paracelsus der Leib Gehäuse und Fenster zugleich, transparent wie ein Glas, eine Öffnung ins All, ein Medium aber auch, das wir nur noch als gebrochen, geschwächt, getrübt erfahren, das daher zeitlebens will geläutert werden, um zur Reife zu kommen, seinen Sinn zu finden.

Und so entfaltet Paracelsus denn auch immer wieder diese so gigantischen, dramaturgisch bewegten Landschaften, in denen die Elemente der Welt ihr Spiel mit dem Menschen beginnen und bestimmen und beenden. «Denn die Elemente und der Mensch sind näher und gefreundeter denn Mann und Weib. Das macht die Konkordanz der Union, so die Elemente gegen den Menschen haben wie auch die Dissonanz, so Frau und Mann gegeneinander haben» (XI, 178). Alle Elemente der Welt, sie sind ja auch im Menschen, und alles ist eine Aktion, eine einzige elementare Interaktion, und genau so dramatisch inszeniert wie in der Dualunion von Mann und Frau.

Um diesen leibhaftigen «status hominis», der allein «die Höhe des Menschen antrifft», noch näher zu charakterisieren, spricht Paracelsus von der «Ehe zwischen Seele und Leib», die erst das geistige Zusammenspiel unserer Existenz ermöglicht. Ein solches wahrhaft «spirituelles» Dasein aber wäre 
gar nicht denkbar ohne den Leib. Denn «Leib und Geist sind ein Ding»! Leib und Geist, sie beide können nur lebendig gedacht sein; «denn die zwei geben einen Menschen» $(\mathrm{X}, 651)$. Und noch einmal und gesteigert: «Der Leib, die Seel', der Geist, die machen einen Menschen. So die drei beinander sind, so ist das das Leben»!

Der gebildete Leib aber, das ist nun auch der ausgewachsene Leib, «der sein Selbst empfindet». Denn «also ist die Art des Lichts der Natur, dass sie in die Wiege eingehet, dass sie mit Ruten eingeschlagen wird, dass sie mit dem Haar herzu gezogen wird und gehet hinein dermassen, dass sie kleiner denn das Senfkorn ist und wachset über den Senfbaum» (VIII, 211). In diesem Baum nun nisten die Vögel, was wiederum nichts anderes zu bedeuten hat, als «daß das Junge in uns komme, das im Alter gross wird und also gross, dass nicht allein der Mensch für sich selbst ist, sondern auch für alle andern». Drum solle auch der Mensch werden - wachsen wie ein Baum - und «also das Exempel Christi erfüllen»(VIII, 212).

Auch in seinen theologischen Traktaten bringt Paracelsus immer wieder das gleiche Bild, so in einem Kommentar zum Psalm 103, wo wir lesen: «Wir alle sind Senfkörner, drum sollen wir wachsen, auf dass, so wir zu unserem Alter kommen, die Engel Gottes in uns wohnen und nisten». Und noch einmal: Denn «unsere Werke folgen uns nach, das ist: dass wir werden darin blühen und wachsen wie ein Senfkorn, auf Erden klein sein, aber im Himmel werden unsre Engel in uns wohnen» (I, 319). Dann erst, «dann werden wir im Alter die Wahrheit Gottes sehen, unser Geblüh und unser Sprossen» (In Ps. 91).

Und so liegt denn auch der Sinn aller Natur, aller sinnlichen Bindung allein in der leibhaftigen Bildung der Welt. Dermassen ward der Mensch «in die Gestalt geschaffen, dass alle Magnalia naturae durch ihn mögen sichtiglich geschehen» (XII, 57). Alle natürlichen Mysterien der Schöpfung, sie wollen offenbar werden, was ohne den Menschen nicht geschehen könnte.

Was an geistigem Impetus des Theophrastus von Hohenheim weiterwirkte, war denn auch nicht die begriffliche Nomenklatur oder das technische Instrumentarium diagnostischer oder therapeutischer Art, was weiterwirkte, war eher sein philosophischer Geist und daraus abgeleitet ein ethisches Prinzip, das alles ärztliche Handeln zu verbinden wusste mit einem omnivalenten Kulturauftrag an der Welt.

Hierzu abschliessend noch ein ganz kurzer Exkurs, der uns einstimmen könnte in das so grossartige Konzept einer Restitution der Natur aus dem 
Geiste, einer Verklärung der Welt im Licht der Natur. Diese längst nicht erschlossenen Texte zu einer paracelsischen Eschatologie erinnern mich aber auch an die dunklen Worte, die Karl Kraus einmal über den dunklen Lichtenberg geprägt hat, wo er von ihm sagt: Er «gräbt tiefer als irgendeiner, aber er kommt nicht wieder hinauf. Er redet unter der Erde. Nur wer selbst tief gräbt, hört ihn. Und man muss schon sehr tief an die Quellen graben, um die dunklen Worte zu hören, zu verstehen.»

Ist sie dann aber da, die «Stunde der Zeit», ist das Gegenwärtige «zeitig» geworden, in uns präsent, dann - so Paracelsus - wird es einfach «heraus müssen wie ein Kind aus dem Bauch seiner Mutter. Was also heraus gehet, das ist fruchtbar und gut». Auffliegen wie ein Adler sollte alsdann der Mensch, um in die «andre Geburt» zu gelangen. Von Natur aus brennt ja schon unser leiblich Leben dahin wie in einem Verbrennungsprozess: «Der Leib ist das Holz, hat sein Feuer in sich und seine Zeit und Stund'». Alle Leiblichkeit ist ja nur Symbol für des Lebens Sinn: «Also im Leib: Wir grünen für und für und haben viel tausenderlei Gesundheit» (IV, 320).

Einmal aber muss sie dann kommen, die Stund', und es wird kommen die Zeit, da wir alle ohn' End' werden wandeln im Angesicht Gottes, «unter welchem kein Arges, kein Übel mehr ist, kein Winter noch rauhe aquilonische Luft, sondern alle Milde wird sein, wie es niemand mag sagen». Dann ist gekommen die Zeit der grossen Ernte, ein «aureum saeculum», jene güldene Zeit, die Paracelsus gepriesen hat als den «Mai der Welt», in dem alles gesundet zum «salus» einer blühenden Natur.

Von einem irdischen Paradies ist jetzt die Rede, das anbrechen werde, sobald unser «zerblendet Herz», vom Mammon befleckt und verflucht, von diesem Gift wird gereinigt werden. Wenn dann der Mammon ausgerottet ist, und alles, was an ihm hängt, «alsdann wird Gott, der Herr, ein neu Jerusalem bauen» (II/VII, 91).

Was den Menschen danach erwartet, wird nur noch angedeutet in Gleichnissen. «Alsdann, wenn der Körper rein und superfein ist, das ist, glorifizieret, von allem Wust und Unrat geschieden, jetzt steigt er auf zu den Himmeln, zu dem Tisch, den uns Gott, der himmlische Vater, bereitet hat, bei seinem Sohne zu essen» (II/I, 311). Alsdann werden dort singen die Heiligen, das sind die Armen, aus vollem Herzen. «Und der Herr wird selber regieren die Welt, der Herr, der Gott in Sion ist» (II/VII, 89). Er hat gebauet das Ewige Jerusalem, «und Er wird das Regiment behalten von Geschlecht zu Geschlecht»! 


\section{Literaturhinweise}

* Zitiert wird - unter leichter Modernisierung - mit lateinischer Bandzahl und arabischer Seitenangabe nach: Theophrast von Hohenheim, gen. Paracelsus: Sämtliche Werke. Hrsg. Karl Sudhoff. Bde. I-XIV. Berlin, München 1922-1933.

Fischer, Hans: Die kosmologische Anthropologie des Paracelsus als Grundlage seiner Medizin. Verhdl. Naturforsch. Ges. Basel 52 (1940/41) 267-317.

Geerk, Frank: Paracelsus - Arzt unserer Zeit. Leben, Werk und Wirkungsgeschichte des Theophrastus von Hohenheim. Zürich 1992.

Goldammer, Kurt: Der göttliche Magier und die Magierin Natur. Religion, Naturmagie und die Anfänge der Naturwissenschaft vom Spätmittelalter bis zur Renaissance. Stuttgart 1991.

- Aufgaben der Paracelsusforschung. In: Parerga Paracelsica. Hrsg. J. Telle. Stuttgart 1991, S.1-26.

Honnefelder, Ludger (Hrsg.): Natur als Gegenstand der Wissenschaften. Freiburg, München 1991.

Joël, Karl: Der Ursprung der Naturphilosophie aus dem Geiste der Mystik. Basel 1913.

Locher, Hans: Theophrastus Paracelsus Bombastus von Hohenheim, der Luther der Medizin und unser grösster Schweizerarzt. Eine Denkschrift auf die Feier des Züricher Jubilarfestes zur Würdigung vaterländischer Verdienste in jedem gebildeten Kreise. Zürich 1851.

Milt, Bernhard: Paracelsus und Zürich. Vierteljahresschrift der Naturforschenden Gesellschaft 86 (1941) 331.

Pagel, Walter: Das medizinische Weltbild des Paracelsus und seine Zusammenhänge mit Neuplatonismus und Gnosis. Wiesbaden 1962.

Paracelsus: Vom Licht der Natur und des Geistes. Eine Auswahl. Hrsg. Kurt Goldammer. Stuttgart 1960.

- Das Licht der Natur. Philosophische Schriften. Hrsg. Rolf Löther und Siegfried Wollgast. Leipzig 1973.

- Vom gesunden und seligen Leben. Ausgewählte Texte. Hrsg. Rolf Löther und Siegfried Wollgast. Leipzig 1991.

Schipperges, Heinrich: Paracelsus. Der Mensch im Licht der Natur. Stuttgart 1974.

- Vom Licht der Natur im Weltbild des Paracelsus. Scheidewege 6 (1976) 30-48.

- Die Entienlehre des Paracelsus. Aufbau und Umriss seiner Theoretischen Pathologie. Berlin, Heidelberg, New York 1988.

Telle, Joachim (Hrsg.): Parerga Paracelsica. Paracelsus in Vergangenheit und Gegenwart. Stuttgart 1991.

Zanier, Giancarlo. L'espressione e l'immagine: introduzione a Paracelso. Triest 1988.

Zekert, Otto : Paracelsus. Europäer im 16. Jahrhundert. Stuttgart, Berlin, Köln, Mainz 1968.

Prof. Dr. H. Schipperges

Schriesheimer Strasse 59

D-69221 Dossenheim 